\title{
Condensed forms for skew-Hamiltonian/Hamiltonian pencils
}

\author{
Christian Mehl ${ }^{\dagger}$
}

\begin{abstract}
In this paper we consider real or complex skew-Hamiltonian/Hamiltonian pencils $\lambda S-H$, i.e., pencils where $S$ is a skew-Hamiltonian and $H$ is a Hamiltonian matrix. These pencils occur for example in the theory of continuous time, linear quadratic optimal control problems. We reduce these pencils to canonical and Schur-type forms under structure-preserving transformations, i.e., $J$-congruence-transformations $(\lambda S-H) \mapsto-J P^{*} J(\lambda S-H) P$, where $P$ is non-singular or unitary.
\end{abstract}

Keywords: Hamiltonian matrices, skew-Hamiltonian matrices, matrix pencils, Schur forms, canonical forms, Riccati equations, descriptor systems, optimal control

AMS classification: 15A21, 15A22, 15A57, 93B25

\section{Introduction}

The motivation for the study of pencils $\lambda S-H$, where $S$ is a skew-Hamiltonian and $H$ is a Hamiltonian matrix, comes mainly from the linear quadratic optimal control problem; see [10], [13], [14], and the references therein. This is the problem of minimizing the cost functional

$$
\frac{1}{2} \int_{t_{0}}^{\infty}\left(y(t)^{*} Q y(t)+u(t)^{*} R u(t)\right) d t
$$

subject to the dynamics

$$
\begin{aligned}
E \dot{x}(t) & =A x(t)+B u(t), \\
x\left(t_{0}\right) & =x_{0}, \\
y(t) & =C x(t),
\end{aligned}
$$

where $A, E \in \mathbb{C}^{n \times n}, Q \in \mathbb{C}^{p \times p}, B \in \mathbb{C}^{n \times m}, C \in \mathbb{C}^{p \times n}, R \in \mathbb{C}^{m \times m}, B$ with full column rank, $C$ with full row rank, $Q$ Hermitian, and $R$ Hermitian positive definite. Solutions of (1) can be obtained via the solution of the generalized continuous algebraic Riccati equation

$$
C^{*} Q C+A^{*} X E+E^{*} X A-\left(B^{*} X E\right)^{*} R^{-1}\left(B^{*} X E\right)=0,
$$

\footnotetext{
${ }^{\dagger}$ Fakultät für Mathematik, Technische Universität Chemnitz, D-09107 Chemnitz, Germany (mehl@mathematik.tu-chemnitz.de).
} 
or directly via the computation of deflating subspaces of the pencil

$$
\lambda S_{0}-H_{0}=\lambda\left[\begin{array}{cc}
E & 0 \\
0 & E^{*}
\end{array}\right]-\left[\begin{array}{cc}
A & B R^{-1} B^{*} \\
C^{*} Q C & -A^{*}
\end{array}\right] ;
$$

see again [10], [13], [14], and the references therein.

From (5) we obtain immediately that $S_{0}$ is a skew-Hamiltonian and $H_{0}$ is a Hamiltonian matrix.

Definition 1 Let $J_{n}:=\left[\begin{array}{cc}0 & I_{n} \\ -I_{n} & 0\end{array}\right]$. (We drop the index $n$ if the dimension is clear from the context.)

1. A matrix $H \in \mathbb{C}^{2 n \times 2 n}$ is called Hamiltonian if

$$
H J+J H^{*}=0 .
$$

The set of Hamiltonian matrices is denoted by $\mathcal{H}_{2 n}$.

2. A matrix $S \in \mathbb{C}^{2 n \times 2 n}$ is called skew-Hamiltonian if

$$
S J-J S^{*}=0 .
$$

The set of skew-Hamiltonian matrices is denoted by $\mathcal{S H}_{2 n}$.

3. A pencil $\lambda S-H \in \mathbb{C}^{2 n \times 2 n}$ is called skew-Hamiltonian/Hamiltonian if $S$ is skewHamiltonian and $H$ is Hamiltonian.

The set of Hamiltonian matrices and the set of skew-Hamiltonian matrices have a special algebraic structure. The first is a Lie algebra (see e.g. [21]) and the latter is a Jordan algebra (see [1]). If the matrices under consideration are complex, there exists a vector space isomorphism between these two algebras.

Lemma 2 The map $A \mapsto i A$ is a vector space isomorphism between $\mathcal{H}_{2 n}$ and $\mathcal{S H}_{2 n}$.

Proof. The proof follows directly from the definition.

If $E$ in (5) is the identity matrix, then the study of the pencil $\lambda S_{0}-H_{0}$ reduces to the study of a Hamiltonian matrix. Condensed forms for Hamiltonian matrices have been extensively studied in recent years; see,e.g., [2], [3], [4], [11], [17], or [5] for a more general approach concerning elements from classical Lie or Jordan algebras. There also have been extensive studies concerning symplectic matrices and symplectic pencils that occur in the context of the discrete algebraic Riccati equation corresponding to (5); see, e.g., [6], [11], $[22]$.

If $E$ in (5) is nonsingular, then it is well known that

$$
S_{0}^{*}\left[\begin{array}{cc}
0 & E^{-*} \\
-E^{-1} & 0
\end{array}\right] H_{0}=-H_{0}^{*}\left[\begin{array}{cc}
0 & E^{-*} \\
-E^{-1} & 0
\end{array}\right] S_{0} ;
$$


i.e., $\lambda\left(i S_{0}\right)-H_{0}$ is an $i\left[\begin{array}{cc}0 & E^{-*} \\ -E^{-1} & 0\end{array}\right]$-self-adjoint pencil or in the real case $\lambda S_{0}-H_{0}$ is a $i\left[\begin{array}{cc}0 & E^{-*} \\ -E^{-1} & 0\end{array}\right]$-skew-symmetric pencil in the sense of [10], where canonical forms for these kinds of pencils were discussed. Obviously this terminology cannot be used if $E$ in (5) is singular. But in recent years, there has been great interest in the singular case; see [13] and the references therein. Therefore we use in this paper the concept of skew-Hamiltonian/Hamiltonian pencils. The case that $E$ is singular is included here.

In the following we will look for condensed forms, i.e., forms from which the eigenvalues of the pencil can be read off in a simple way. For an arbitrary pencil these are the Kronecker canonical form (see [8]) that yields the eigenvalues and eigenvectors and the (generalized) Schur form (see [9]) that yields the eigenvalues and a nested set of invariant subspaces and that is obtained by a transformation $(\lambda A-B) \mapsto U(\lambda A-B) V$, where $U$ and $V$ are unitary matrices. Following the notation in [13] we use the term "Kronecker canonical form" also in the case of regular pencils, but we note that in this case the canonical form is due to Weierstraß; see [8].

The following Lemma is helpful in finding out what kind of structure-preserving transformations we may use to obtain corresponding forms for skew-Hamiltonian/ Hamiltonian pencils.

Lemma 3 The map $H \mapsto J H$ is a bijection between $\mathcal{H}_{2 n}$ and the set of $2 n \times 2 n$ Hermitian matrices.

Proof. The proof follows directly from the definitions.

The general structure-preserving equivalence transformations for Hermitian matrices are the congruence transformations $A \mapsto P^{*} A P$, where $P$ is nonsingular. Lemma 3 implies that the transformations $H \mapsto-J P^{*} J H P$, where $P$ is nonsingular, preserve the Hamiltonian structure. We will call these transformations $J$-congruence transformations by analogy to the congruence transformations. More general are the transformations $H \mapsto \mu J P^{*} J H P$, where $P$ is nonsingular and $\mu \in \mathbb{R}$. These transformations are the analogue of the $\mu$ congruence transformations $A \mapsto \mu P^{*} A P$ (see also [16] for related $\mu$-symplectic transformations).

\section{Definition 4}

1. Two matrices $A, B \in \mathbb{C}^{2 n \times 2 n}$ are called $J$-congruent if there exists a nonsingular matrix $P \in \mathbb{C}^{n \times n}$ such that

$$
-J P^{*} J A P=B
$$

2. Two pencils $\lambda A-B, \lambda C-D \in \mathbb{C}^{2 n \times 2 n}$ are called $J$-congruent if there exists a nonsingular matrix $P \in \mathbb{C}^{n \times n}$ such that

$$
-J P^{*} J(\lambda A-B) P=\lambda C-D .
$$

Analogously we define $\mu$ - $J$-congruence. We easily obtain the following lemma. 
Lemma 5 Let $S \in \mathbb{C}^{2 n \times 2 n}$ be skew-Hamiltonian, $H \in \mathbb{C}^{2 n \times 2 n}$ Hamiltonian, $P \in \mathbb{C}^{2 n \times 2 n}$, and let $\mu \in \mathbb{R}$. Then

1. $\mu J P^{*} J S P$ is skew-Hamiltonian,

2. $\mu J P^{*} J H P$ is Hamiltonian,

3. $\mu J P^{*} J(\lambda S-H) P$ is skew-Hamiltonian/Hamiltonian.

Proof. The proof follows directly from the definitions.

If $\lambda S-H$ is a skew-Hamiltonian/Hamiltonian pencil, then $\lambda(i J S)-(J H)$ is a Hermitian pencil in the sense of [19], where a canonical form for Hermitian pencils is given. In the theory of Hermitian pencils this form is the analogue of the Kronecker canonical form in the theory of general matrix pencils. The numerical computation of the Kronecker canonical form is in general ill-conditioned (see, e.g., [9]) in contrast to the computation of the Schur form (see again [9]). But for a Hermitian matrix the Schur form and the Jordan canonical from coincide; i.e., a triangular form of a Hermitian pencil would be diagonal. Thus, the problem of finding a triangular form for a Hermitian pencil under unitary congruence transformations is equivalent to the problem of the simultaneous diagonalization of two Hermitian matrices. This is possible if and only if the matrices commute; see [18].

In contrast to this, the concept of skew-Hamiltonian/Hamiltonian pencils enables us to obtain both a canonical form and a Schur-type form under $J$-congruence. After stating some preliminary results in section 2 we discuss this canonical form in section 3 . In regard of our interest in finding eigenvalues we restrict our examination to regular pencils. In section 4 we discuss under which conditions it is possible to obtain a structured Schur form under unitary $J$-congruence transformations, i.e., $J$-congruence transformations, where the matrix $P$ in $(8)$ is unitary. We will call this form a $J$-Schur form. Since in general not every skew-Hamiltonian/Hamiltonian pencil has a $J$-Schur form, we present in section 5 a closely related form that is almost a Schur form and that is obtained by using also some nonunitary $J$-congruence transformations.

Throughout the paper we use the following notation and expressions:

1. By the direct sum of two square matrices $A, B$, we mean the matrix $\left[\begin{array}{ll}A & 0 \\ 0 & B\end{array}\right]$. Analogously we define the direct sum of square pencils.

2. By $\operatorname{Span}\left(x_{1}, \ldots, x_{m}\right)$ we denote the subspace of $\mathbb{C}^{k}$ spanned by the vectors $x_{1}, \ldots, x_{m}$.

3. By $e_{i}$ we denote the $i$ th unit vector.

4. By $\sim_{c}$ we denote the equivalence relation by congruence.

5. Let $\lambda A-B \in \mathbb{C}^{n \times n}$ be a regular pencil. Introducing homogeneous parameters, $\alpha A-\beta B$, see [8], the eigenvalues of the pencils $\alpha A-\beta B$ can be represented by pairs $(\alpha, \beta) \in \mathbb{C}^{2} \backslash\{0\}$, such that

$$
\alpha A x-\beta B x=0 \text { for an } x \in \mathbb{C}^{n} \backslash\{0\} .
$$


Obviously $(t \alpha, t \beta)$ represents the same eigenvalue for all $t \in \mathbb{C} \backslash\{0\}$; thus, we denote them by $\lambda=\frac{\alpha}{\beta}$ if $\beta \neq 0$. Pairs $(\alpha, 0), \alpha \neq 0$ represent the eigenvalue infinity of $\alpha A-\beta B$ that we will denote by $\infty$. We note that we always include the eigenvalue $\infty$ when we are talking about real or about purely imaginary eigenvalues.

\section{Preliminaries}

We first review some properties of the pencils under consideration.

Lemma 6 Let $\lambda S-H$ be a skew-Hamiltonian/Hamiltonian pencil. If $\lambda_{0}$ is an eigenvalue of $\lambda S-H$, then so is $-\lambda_{0}^{*}$ with the same partial multiplicities.

Proof. Since $S$ is skew-Hamiltonian and $H$ is Hamiltonian we find that

$$
J(\lambda S-H) J=-\lambda S^{*}-H^{*}
$$

i.e., $\lambda S-H$ and $-\lambda S^{*}-H^{*}$ are equivalent. Thus, these two pencils have the same Kronecker canonical form.

To every block associated with an eigenvalue $\lambda_{0}$ with nonzero real part in the Kronecker canonical form, we find by Lemma 6 a paired block of the same size associated with the eigenvalue $-\lambda_{0}^{*}$. In general we have no pairing of blocks for the purely imaginary eigenvalues.

In the following we will use the concept of principle vectors of matrix pencils see ([7], [8], and [13]) that is analogous to the concept of principle vectors of matrices or the concept of Jordan chains. Since the chains of principle vectors lead in the pencil case to the Kronecker canonical form, we call them Kronecker chains.

Definition 7 Let $\lambda A-B \in \mathbb{C}^{n \times n}$ be a regular pencil.

1. A Kronecker chain associated with a finite eigenvalue $\lambda_{0}$ of $\lambda A-B$ is a tuple $\left(x_{1}, \ldots, x_{m}\right)$ of vectors from $\mathbb{C}^{n} \backslash\{0\}$ such that

$$
\begin{aligned}
\lambda_{0} A x_{1} & =B x_{1} \\
\text { and } \quad \lambda_{0} A x_{i}-B x_{i} & =-A x_{i-1} \quad \text { for } i=2, \ldots, m .
\end{aligned}
$$

2. A Kronecker chain associated with the eigenvalue $\infty$ of $\lambda A-B$ is a tuple $\left(y_{1}, \ldots, y_{k}\right)$ of vectors from $\mathbb{C}^{n} \backslash\{0\}$ such that

$$
\begin{aligned}
A y_{1} & =0 \\
\text { and } A y_{i} & =B y_{i-1} \quad \text { for } i=2, \ldots, k .
\end{aligned}
$$

It is clear that if the Kronecker chains in Definition 7 are of maximal length, we find a block $\lambda_{0} I_{m}-J_{m}(\lambda)$ in the Kronecker canonical form of $\lambda A-B$, whose associated deflating subspace is spanned by $x_{1}, \ldots, x_{m}$ and a block $\lambda J_{k}(0)-I_{k}$, whose associated deflating 
subspace is spanned by $y_{1}, \ldots, y_{k}$. Here $J_{l}(\mu)$ denotes an $l \times l$ Jordan block with eigenvalue $\mu$ :

$$
J_{l}(\mu)=\left[\begin{array}{ccccc}
\mu & 1 & 0 & \ldots & 0 \\
0 & \mu & 1 & \ddots & \vdots \\
\vdots & \ddots & \ddots & \ddots & 0 \\
\vdots & & \ddots & \ddots & 1 \\
0 & \ldots & \ldots & 0 & \mu
\end{array}\right]
$$

Furthermore, we have the following property.

Lemma 8 Let $\lambda A-B \in \mathbb{C}^{n \times n}$ be a regular pencil. If $\left(x_{1}, \ldots, x_{m}\right)$ is a Kronecker chain associated with the eigenvalue 0 of $\lambda A-B$, then $\left(x_{1}, \ldots, x_{m}\right)$ is a Kronecker chain associated with the eigenvalue $\infty$ of $\lambda B-A$.

Proof. The proof follows directly from Definition 7 .

The structure of skew-Hamiltonian/Hamiltonian pencils leads to special properties of the Kronecker chains.

Lemma 9 Let $\lambda S-H \in \mathbb{C}^{2 n \times 2 n}$ be a regular skew-Hamiltonian/Hamiltonian pencil. Furthermore let $\left(x_{1}, \ldots, x_{m}\right)$ be a Kronecker chain associated with the eigenvalue $\lambda_{0} \in \mathbb{C} \cup\{\infty\}$ and $\left(y_{1}, \ldots, y_{l}\right)$ a Kronecker chain associated with the eigenvalue $\mu_{0} \in \mathbb{C} \cup\{\infty\}$, where $\mu_{0} \neq-\lambda_{0}^{*}$. Then for all $i, j$, where $1 \leq i \leq l$ and $1 \leq j \leq m$, we have

$$
y_{i}^{*} J S x_{j}=y_{i}^{*} J H x_{j}=0 .
$$

Proof. The proof proceeds via induction on $k=i+j$.

1. $k=2$, i.e., $i=j=1$ : Since $\mu \neq-\lambda^{*}$, at most one of these eigenvalues is infinite. Thus, we may assume without loss of generality (w.l.o.g.) that $\lambda \neq \infty$.

(a) In the case $\mu=\infty$, we have $S y_{1}=0$. Since $S$ is skew-Hamiltonian, we have

$$
y_{1}^{*} J S x_{1}=y_{1}^{*} S^{*} J x_{1}=0 .
$$

Furthermore, we obtain from $\lambda S x_{1}=H x_{1}$, that

$$
y_{1}^{*} J H x_{1}=\lambda y_{1}^{*} J S x_{1}=0 .
$$

(b) In the case $\mu \neq \infty$, we have

$$
\mu S y_{1}=H y_{1} .
$$

Noting that $S$ is skew-Hamiltonian and $H$ is Hamiltonian, we see that this implies

$$
\begin{aligned}
\mu^{*} y_{1}^{*} J S x_{1} & =\left(\mu x_{1}^{*} J S y_{1}\right)^{*} \\
& =\left(x_{1}^{*} J H y_{1}\right)^{*} \\
& =-y_{1}^{*} J H x_{1} .
\end{aligned}
$$


Since we also have $\lambda y_{1}^{*} J S x_{1}=y_{1}^{*} J H x_{1}=0$, we obtain

$$
\left(\lambda+\mu^{*}\right) y_{1}^{*} J S x_{1}=0 .
$$

Thus, we obtain $y_{1}^{*} J S x_{1}=0$, since $\mu \neq-\lambda^{*}$, and therefore also $y_{1}^{*} J H x_{1}=0$.

2. $k \Rightarrow k+1$ : Suppose that we have already proved (13) for all $i, j$, where $i \leq l, j \leq m$ and $i+j=k$. Obviously it is sufficient to show that

$$
y_{i}^{*} J S x_{j+1}=y_{i}^{*} J H x_{j+1}=0
$$

for all $i, j$, where $i \leq l, j<m$ and $i+j=k$. Now assume again w.l.o.g., that $\lambda \neq \infty$ and let $y_{0}:=0$.

(a) In the case $\mu=\infty$ we have $S y_{i}=H y_{i-1}$. Note that this holds also in the case $i=1$, since $y_{0}=0$. By this or in the case $i>1$ by induction, we obtain

$$
\begin{aligned}
y_{i}^{*} J S x_{j+1} & =y_{i}^{*} S^{*} J x_{j+1} \\
& =y_{i-1}^{*} H^{*} J x_{j+1} \\
& =0 .
\end{aligned}
$$

From $\lambda S x_{j+1}-H x_{j+1}=-S x_{j}$ we also obtain

$$
y_{i}^{*} J H x_{j+1}=\lambda y_{i}^{*} J S x_{j+1}+y_{i}^{*} J S x_{j}=0 .
$$

(b) In the case $\mu \neq \infty$ we have $\mu S y_{i}-H y_{i}=-S y_{i-1}$. Since $x_{j+1}^{*} J S y_{i-1}=0$, this yields

$$
\begin{aligned}
\mu^{*} y_{i}^{*} J S x_{j+1} & =\left(\mu x_{j+1}^{*} J S y_{i}\right)^{*} \\
& =\left(x_{j+1}^{*} J H y_{i}\right)^{*} \\
& =-y_{i}^{*} J H x_{j+1}
\end{aligned}
$$

once more using the fact that $S$ is skew-Hamiltonian and that $H$ is Hamiltonian. Noting that $y_{i}^{*} J S x_{j}=0$, we also have

$$
\lambda y_{i}^{*} J S x_{j+1}=y_{i}^{*} J H x_{j+1} .
$$

Therefore, we obtain

$$
\left(\lambda+\mu^{*}\right) y_{i}^{*} J S x_{j+1}
$$

and finally

$$
y_{i}^{*} J S x_{j+1}=y_{i}^{*} J H x_{j+1}=0 .
$$

Lemma 10 Let $\lambda S-H \in \mathbb{C}^{2 n \times 2 n}$ be a regular skew-Hamiltonian/Hamiltonian pencil. Furthermore let $\left(x_{1}, \ldots, x_{m}\right)$ be a Kronecker chain associated with the eigenvalue $\lambda \in \mathbb{C} \cup\{\infty\}$ and $\left(y_{1}, \ldots, y_{l}\right)$ a Kronecker chain associated with the eigenvalue $\mu$, where $\mu=-\lambda^{*}$. Then for all $i, j$ where $1 \leq i \leq l, 1 \leq j \leq m$ and $i+j \leq \max (l, m)$, we have

$$
y_{i}^{*} J S x_{j}=y_{i}^{*} J H x_{j}=0 .
$$


Proof. If $\lambda=\infty$, then Lemma 8 implies that $\left(x_{1}, \ldots, x_{m}\right)$ and $\left(y_{1}, \ldots, y_{l}\right)$ are Kronecker chains of $\lambda H-S$ associated with the eigenvalue zero. This also holds for the skewHamiltonian/Hamiltonian pencil $\lambda(i H)-(i S)$. Thus, it remains to prove the assertion for the case that $\lambda \neq \infty$.

1. First of all we prove that for all $i, j$, where $1 \leq i \leq l$ and $0 \leq j<m$, we have

$$
y_{i}^{*} J S x_{j}=-y_{i-1}^{*} J S x_{j+1} \text { and } y_{i}^{*} J H x_{j}=-y_{i-1}^{*} J H x_{j+1},
$$

where $x_{0}:=0$ and $y_{0}:=0$. Since $\left(x_{1}, \ldots, x_{m}\right)$ and $\left(y_{1}, \ldots, y_{l}\right)$ are Kronecker chains we have

$$
\lambda S x_{j+1}-H x_{j+1}=-S x_{j} \text { and } \quad-\lambda^{*} S y_{i}-H y_{i}=-S y_{i-1} .
$$

Note that this is also true for $i=1$ and $j=0$. From (15) and since $S$ is skew-Hamiltonian and $H$ is Hamiltonian, we obtain

$$
\begin{aligned}
-y_{i-1}^{*} J S x_{j+1} & =-y_{i-1}^{*} S^{*} J x_{j+1} \\
& =-\lambda y_{i}^{*} S^{*} J x_{j+1}-y_{i}^{*} H^{*} J x_{j+1} \\
& =-\lambda y_{i}^{*} J S x_{j+1}+y_{i}^{*} J H x_{j+1} \\
& =y_{i}^{*} J S x_{j}
\end{aligned}
$$

and analogously

$$
\begin{aligned}
-y_{i-1}^{*} J H x_{j+1} & =-\lambda y_{i-1}^{*} J S x_{j+1}-y_{i-1}^{*} J S x_{j} \\
& =\lambda y_{i}^{*} J S x_{j}-y_{i-1}^{*} J S x_{j} \\
& =\left(\lambda y_{i}^{*} S^{*}-y_{i-1}^{*} S^{*}\right) J x_{j} \\
& =-y_{i}^{*} H^{*} J x_{j} \\
& =y_{i}^{*} J H x_{j} .
\end{aligned}
$$

2. Now assume w.l.o.g. that $l=\max (l, m)$. Since $i+j \leq l$ and $x_{0}=0$ we obtain by (14) that

$$
y_{i}^{*} J S x_{j}=(-1)^{j} y_{i+j}^{*} J S x_{0}=0
$$

and

$$
y_{i}^{*} J H x_{j}=(-1)^{j} y_{i+j}^{*} J H x_{0}=0 .
$$

\section{A canonical form under $J$-congruence}

In this section we present a canonical form for skew-Hamiltonian/Hamiltonian pencils under $J$-congruence. In the study of skew-Hamiltonian/Hamiltonian pencils this form is the analogue of the Kronecker canonical form in the study of general matrix pencils. 
Theorem 11 Let $\lambda S-H \in \mathbb{C}^{2 n \times 2 n}$ be a regular skew-Hamiltonian/Hamiltonian pencil. Then there exists a nonsingular matrix $P \in \mathbb{C}^{2 n \times 2 n}$ such that

$$
\begin{aligned}
& -J P^{*} J(\lambda S-H) P \\
= & \lambda\left[\begin{array}{cccc}
S_{11} & 0 & 0 & 0 \\
0 & S_{22} & 0 & S_{24} \\
0 & 0 & S_{11}^{*} & 0 \\
0 & S_{42} & 0 & S_{22}^{*}
\end{array}\right]-\left[\begin{array}{cccc}
H_{11} & 0 & 0 & 0 \\
0 & H_{22} & 0 & H_{24} \\
0 & 0 & -H_{11}^{*} & 0 \\
0 & H_{42} & 0 & -H_{22}^{*}
\end{array}\right],
\end{aligned}
$$

where

1. $\lambda S_{11}-H_{11}$ is a pencil in Kronecker canonical form and has only eigenvalues with positive real part.

2. $\lambda \hat{S}-\hat{H}:=\lambda\left[\begin{array}{ll}S_{22} & S_{24} \\ S_{42} & S_{22}^{*}\end{array}\right]-\left[\begin{array}{cc}H_{22} & H_{24} \\ H_{42} & -H_{22}^{*}\end{array}\right]$ is a skew-Hamiltonian/Hamiltonian pencil, such that $J(\lambda \hat{S}-\hat{H})$ is the direct sum of blocks of the form

$$
\lambda \varepsilon\left[\begin{array}{lll}
0 & & i \\
& \cdot & \\
& \cdot & \\
i & & 0
\end{array}\right]-\varepsilon\left[\begin{array}{llll}
0 & & & \mu \\
& & \cdot & 1 \\
& \cdot & \cdot & \\
\mu & 1 & & 0
\end{array}\right] \in \mathbb{C}^{k \times k}
$$

or

$$
\lambda \varepsilon\left[\begin{array}{llll}
0 & & & 0 \\
& & \cdot & i \\
& \cdot & \cdot & \\
0 & i & & 0
\end{array}\right]-\varepsilon\left[\begin{array}{lll}
0 & & 1 \\
& \cdot & \\
& \cdot & \\
1 & & 0
\end{array}\right] \in \mathbb{C}^{k \times k},
$$

where $\mu$ is real and $\varepsilon= \pm 1$.

Proof.

1. We first prove that $\lambda S-H$ is, up to the permutation of rows and columns, $J-$ congruent to the direct sum of two skew-Hamiltonian/Hamiltonian pencils such that the eigenvalues of one pencil have all nonzero real part and the eigenvalues of the other pencil are all purely imaginary.

Let us consider a basis $\left(x_{1}, \ldots, x_{m}, y_{1}, \ldots, y_{m}, v_{1}, \ldots, v_{2(n-m)}\right)$ of $\mathbb{C}^{2 n}$ consisting of Kronecker chains of $\lambda S-H$ such that $\left(x_{1}, \ldots, x_{m}\right)$ is a basis of the deflating subspace associated with the eigenvalues with positive real part, $\left(y_{1}, \ldots, y_{m}\right)$ is a basis of the deflating subspace associated with the eigenvalues with negative real part, and $\left(v_{1}, \ldots, v_{2(n-m)}\right)$ is a basis of the deflating subspace associated with the purely imaginary eigenvalues. Setting

$$
Q=\left[x_{1}, \ldots, x_{m}, v_{1}, \ldots, v_{n-m}, y_{1}, \ldots, y_{m}, v_{n-m+1}, \ldots, v_{2(n-m)}\right]
$$

we find by Lemma 9 that

$$
\begin{aligned}
& -J Q^{*} J(\lambda S-H) Q \\
= & \lambda\left[\begin{array}{cccc}
\tilde{S}_{11} & 0 & 0 & 0 \\
0 & \tilde{S}_{22} & 0 & \tilde{S}_{24} \\
0 & 0 & \tilde{S}_{11}^{*} & 0 \\
0 & \tilde{S}_{42} & 0 & \tilde{S}_{22}^{*}
\end{array}\right]-\left[\begin{array}{cccc}
\tilde{H}_{11} & 0 & 0 & 0 \\
0 & \tilde{H}_{22} & 0 & \tilde{H}_{24} \\
0 & 0 & -\tilde{H}_{11}^{*} & 0 \\
0 & \tilde{H}_{42} & 0 & -\tilde{H}_{22}^{*}
\end{array}\right],
\end{aligned}
$$


where $\lambda \tilde{S}_{11}-\tilde{H}_{11}$ has only eigenvalues with positive real part and the skew-Hamiltonian/Hamiltonian pencil $\lambda \tilde{S}-\tilde{H}:=\lambda\left[\begin{array}{cc}\tilde{S}_{22} & \tilde{S}_{24} \\ \tilde{S}_{42} & \tilde{S}_{22}^{*}\end{array}\right]-\left[\begin{array}{cc}\tilde{H}_{22} & \tilde{H}_{24} \\ \tilde{H}_{42} & -\tilde{H}_{22}^{*}\end{array}\right]$ has only purely imaginary eigenvalues.

2. For the proof of $(16)$ we note that $J(\lambda(i \tilde{S})-\tilde{H})$ is a Hermitian pencil that has only purely imaginary eigenvalues. Thus, applying Lemma 2 from [19], we find that there exists a nonsingular matrix $X=\left[\begin{array}{ll}X_{11} & X_{12} \\ X_{21} & X_{22}\end{array}\right]$, where $X_{j k} \in \mathbb{C}^{(n-m) \times(n-m)}$, such that $X^{*} J(\lambda \tilde{S}-\tilde{H}) X$ is a direct sum of blocks of the form (17) or (18). Furthermore, we find nonsingular matrices $R, T$ such that $R\left(\lambda \tilde{S}_{11}-\tilde{H}_{11}\right) T$ is in Kronecker canonical form. Hence we obtain (16) by setting

$$
P=Q \cdot\left[\begin{array}{cccc}
R & 0 & 0 & 0 \\
0 & X_{11} & 0 & X_{12} \\
0 & 0 & -T^{*} & 0 \\
0 & X_{21} & 0 & X_{22}
\end{array}\right] \cdot
$$

Note that the blocks of the submatrices $\hat{S}$ and $\hat{H}$ in (16) in general occur in patterns such as

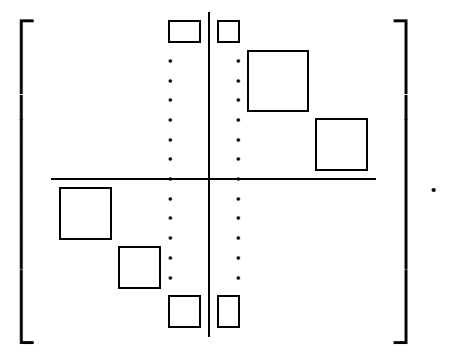

In general it is not possible to reduce the subpencil $\lambda \hat{S}-\hat{H}$ to a direct sum of skewHamiltonian/Hamiltonian pencils that have only one eigenvalue. As an example, consider the pencil

$$
\lambda\left[\begin{array}{ll}
0 & i \\
i & 0
\end{array}\right]-\left[\begin{array}{cc}
0 & 1 \\
-1 & 0
\end{array}\right]
$$

Further reduction is impossible, since the pencil has purely imaginary eigenvalues with odd algebraic multiplicity.

Lemma 12 Let $\lambda S-H \in \mathbb{C}^{2 n \times 2 n}$ be a regular skew-Hamiltonian/Hamiltonian pencil with pairwise distinct eigenvalues $\lambda_{1}, \ldots, \lambda_{k}$ that are all purely imaginary. Then the following statements are equivalent.

1. The eigenvalues $\lambda_{1}, \ldots, \lambda_{k}$ all have even algebraic multiplicity.

2. There exists a nonsingular matrix $P$ such that

$$
-J P^{*} J S P=\left[\begin{array}{ccc|ccc}
S_{11} & & 0 & S_{1, k+1} & & 0 \\
& \ddots & & & \ddots & \\
0 & & S_{k k} & 0 & & S_{k, 2 k} \\
\hline S_{k+1,1} & & 0 & S_{k+1, k+1} & & 0 \\
& \ddots & & & \ddots & \\
0 & & S_{2 k, k} & 0 & & S_{2 k, 2 k}
\end{array}\right]
$$


and

$$
-J P^{*} J H P=\left[\begin{array}{ccc|ccc}
H_{11} & & 0 & H_{1, k+1} & & 0 \\
& \ddots & & & \ddots & \\
0 & & H_{k k} & 0 & & H_{k, 2 k} \\
\hline H_{k+1,1} & & 0 & H_{k+1, k+1} & & 0 \\
& \ddots & & & \ddots & \\
0 & & H_{2 k, k} & 0 & & H_{2 k, 2 k}
\end{array}\right] \text {, }
$$

where $\lambda\left[\begin{array}{cc}S_{i i} & S_{i, k+i} \\ S_{k+i, i} & S_{k+i, k+i}\end{array}\right]-\left[\begin{array}{cc}H_{i i} & H_{i, k+i} \\ H_{k+i, i} & H_{k+i, k+i}\end{array}\right], i=1, \ldots, k$, is a skew-Hamiltonian/Hamiltonian pencil having only one eigenvalue.

Proof. $1 \Rightarrow 2$ : For the deflating subspace associated with $\lambda_{i}$, we find a basis $\left(x_{1}, \ldots, x_{m_{i}}\right)$ consisting of Kronecker chains. Now set

$$
P_{i 1}=\left[x_{1}, \ldots, x_{\frac{m_{i}}{2}}\right] \text { and } P_{i 2}=\left[x_{\frac{m_{i}}{2}+1}, \ldots, x_{m_{i}}\right] .
$$

This is possible, since every eigenvalue has even algebraic multiplicity. Setting

$$
P=\left[P_{11}, \ldots, P_{k 1}, P_{12}, \ldots, P_{k 2}\right]
$$

Lemma 9 implies the required result.

$2 \Rightarrow 1$ : is clear, since the subpencils

$$
\lambda\left[\begin{array}{cc}
S_{i i} & S_{i, k+i} \\
S_{k+i, i} & S_{k+i, k+i}
\end{array}\right]-\left[\begin{array}{cc}
H_{i i} & H_{i, k+i} \\
H_{k+i, i} & H_{k+i, k+i}
\end{array}\right]
$$

have even sizes.

There exists a real analogue to Theorem 11 .

Theorem 13 Let $\lambda S-H \in \mathbb{R}^{2 n \times 2 n}$ be a regular skew-Hamiltonian/Hamiltonian pencil. Then there exists a nonsingular matrix $P \in \mathbb{R}^{2 n \times 2 n}$ such that

$$
\begin{aligned}
& -J P^{*} J(\lambda S-H) P \\
= & \lambda\left[\begin{array}{cccc}
S_{11} & 0 & 0 & 0 \\
0 & S_{22} & 0 & S_{24} \\
0 & 0 & S_{11}^{*} & 0 \\
0 & S_{42} & 0 & S_{22}^{*}
\end{array}\right]-\left[\begin{array}{cccc}
H_{11} & 0 & 0 & 0 \\
0 & H_{22} & 0 & H_{24} \\
0 & 0 & -H_{11}^{*} & 0 \\
0 & H_{42} & 0 & -H_{22}^{*}
\end{array}\right],
\end{aligned}
$$

where

1. $\lambda S_{11}-H_{11}$ is a pencil in real Kronecker canonical form and has only eigenvalues with positive real part. 
2. $\lambda \hat{S}-\hat{H}:=\lambda\left[\begin{array}{ll}S_{22} & S_{24} \\ S_{42} & S_{22}^{*}\end{array}\right]-\left[\begin{array}{cc}H_{22} & H_{24} \\ H_{42} & -H_{22}^{*}\end{array}\right]$ is a skew-Hamiltonian/Hamiltonian pencil such that $J(\lambda \hat{S}-\hat{H})$ is the direct sum of blocks either of the form

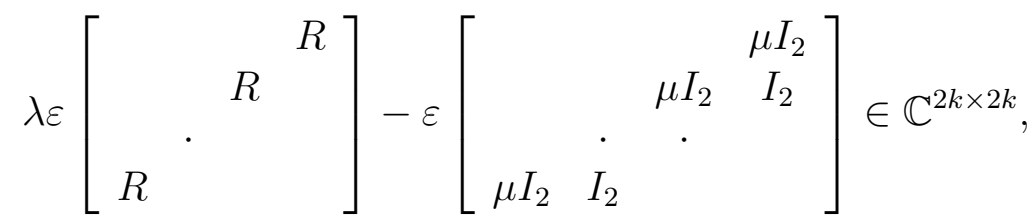

where $\mu>0, R=\left[\begin{array}{cc}0 & -1 \\ 1 & 0\end{array}\right]$ and $I_{2}=\left[\begin{array}{ll}1 & 0 \\ 0 & 1\end{array}\right]$, or of one of the forms

$$
\begin{gathered}
\lambda \varepsilon\left[\begin{array}{ccc}
0 & 0 & 0 \\
0 & 0 & \Delta_{k} \\
0 & -\Delta_{k} & 0
\end{array}\right]-\varepsilon \Delta_{2 k+1}, k \in \mathbb{N}, \\
\lambda\left[\begin{array}{cc}
0 & \Lambda_{k} \\
-\Lambda_{k} & 0
\end{array}\right]-\left[\begin{array}{cc}
0 & \Delta_{k} \\
\Delta_{k} & 0
\end{array}\right], k \text { even, } \\
\lambda \varepsilon\left[\begin{array}{cc}
0 & \Delta_{k} \\
-\Delta_{k} & 0
\end{array}\right]-\varepsilon \Lambda_{2 k}, k \in \mathbb{N},
\end{gathered}
$$

or

$$
\lambda\left[\begin{array}{cc}
0 & \Delta_{k} \\
-\Delta_{k} & 0
\end{array}\right]-\left[\begin{array}{cc}
0 & \Lambda_{k} \\
\Lambda_{k} & 0
\end{array}\right], k \text { odd }
$$

where $\varepsilon= \pm 1$ and furthermore

$$
\Delta_{k}:=\left[\begin{array}{ccc}
0 & & 1 \\
& \cdot & \\
1 & & 0
\end{array}\right] \text { and } \Lambda_{k}:=\left[\begin{array}{cccc}
0 & & & 0 \\
& & \cdot & 1 \\
& \cdot & \cdot & \\
0 & 1 & & 0
\end{array}\right] .
$$

Proof. The proof is similar to the proof of Theorem 11 using results on pencils $\lambda A-B$, where $A$ is skew symmetric and $B$ is symmetric; see, e.g., [20].

Corollary 14 Let $\lambda S-H$ be a real skew-Hamiltonian/Hamiltonian pencil. Then the following conditions apply:

1. Every block of odd size belonging to the eigenvalue zero in the (general) Kronecker canonical form occurs with even multiplicity.

2. Every block of even size belonging to the eigenvalue $\infty$ in the (general) Kronecker canonical form occurs with even multiplicity.

Proof. This follows directly from the structures of the blocks (23) and (25) in the structured canonical form (20). 


\section{The existence of $J$-Schur forms}

As shown in [17] not every Hamiltonian matrix can be reduced by unitary symplectic similarity transformations to a matrix in a Schur-type form that is called Schur-Hamiltonian form in [17] or Hamiltonian Schur form in [13]. This is a Hamiltonian matrix

$$
\left[\begin{array}{cc}
T & R \\
0 & -T^{*}
\end{array}\right]
$$

where $T \in \mathbb{C}^{n \times n}$ is upper triangular and $R \in \mathbb{C}^{n \times n}$ is Hermitian. The analogous Schur-type form for a skew-Hamiltonian matrix is

$$
\left[\begin{array}{cc}
T & R \\
0 & T^{*}
\end{array}\right]
$$

where $T \in \mathbb{C}^{n \times n}$ is upper triangular and $R \in \mathbb{C}^{n \times n}$ is skew-Hermitian. We call matrices of the form (26) or (27) matrices in $J$-Schur form. In [12] and [15] a necessary and sufficient condition for the existence of the Hamiltonian Schur form, resp., $J$-Schur form is presented.

Theorem 15 Let $H \in \mathbb{C}^{2 n \times 2 n}$ be a Hamiltonian matrix and let $\lambda_{1}, \ldots, \lambda_{m}$ be the pairwise distinct purely imaginary eigenvalues of $H$ with algebraic multiplicities $p_{1}, \ldots, p_{m}$. Then the following statements are equivalent:

1. There exists a unitary symplectic matrix $P \in \mathbb{C}^{2 n \times 2 n}$ such that $P H P^{-1}$ is in $J$-Schur form (26).

2. If the columns of $U_{k} \in \mathbb{C}^{2 n \times p_{k}}, k=1, \ldots, m$, form a basis of the invariant subspace associated with $\lambda_{k}$, then

$$
U_{k}^{*} J U_{k} \sim_{c} J_{p_{k} / 2}
$$

Note that (28) implies that the multiplicities $p_{k}$ are even.

Proof. See [12] or [15].

An analogous result holds in the case that $H$ is skew-Hamiltonian.

Theorem 16 Let $S \in \mathbb{C}^{2 n \times 2 n}$ be a skew-Hamiltonian matrix and let $\lambda_{1}, \ldots, \lambda_{m}$ be the pairwise distinct real eigenvalues of $S$ with algebraic multiplicities $p_{1}, \ldots, p_{m}$. Then the following statements are equivalent.

1. There exists a unitary symplectic matrix $P \in \mathbb{C}^{2 n \times 2 n}$ such that $P S P^{-1}$ is in $J$-Schur form (27).

2. If the columns of $U_{k} \in \mathbb{C}^{2 n \times p_{k}}, k=1, \ldots, m$, form a basis of the invariant subspace associated with $\lambda_{k}$, then

$$
U_{k}^{*} J U_{k} \sim_{c} J_{p_{k} / 2}
$$


Proof. The proof follows directly from Lemma 2 and Theorem 15.

We also obtain a similar result for the skew-Hamiltonian/Hamiltonian pencils. For the proof of this result, we need the following lemma.

Lemma 17 Let $P \in \mathbb{C}^{2 n \times 2 n}$. Then there exists a factorization

$$
P=Q \cdot\left[\begin{array}{cc}
R_{11} & R_{12} \\
0 & R_{22}^{*}
\end{array}\right]
$$

where $Q \in \mathbb{C}^{2 n \times 2 n}$ is unitary and $R_{11}, R_{22} \in \mathbb{C}^{n \times n}$ are upper triangular.

Proof. After performing $n$ steps of the QR Householder algorithm (see, e.g., [9]), we find a unitary matrix $Q_{1} \in \mathbb{C}^{n \times n}$ such that

$$
P=Q_{1} \cdot\left[\begin{array}{cc}
R_{11} & \tilde{P}_{12} \\
0 & \tilde{P}_{22}
\end{array}\right],
$$

where $R_{11} \in \mathbb{C}^{n \times n}$ is upper triangular. Then we compute the QL factorization

$$
\tilde{P}_{22}=Q_{2} \cdot R_{22}^{*}
$$

where $Q_{2} \in \mathbb{C}^{n \times n}$ is unitary and $R_{22}^{*} \in \mathbb{C}^{n \times n}$ is lower triangular; see again [9]. Thus, setting $Q=Q_{1} \cdot\left[\begin{array}{cc}I_{n} & 0 \\ 0 & Q_{2}\end{array}\right]$ implies (30).

Theorem 18 Let $\lambda S-H \in \mathbb{C}^{2 n \times 2 n}$ be a skew-Hamiltonian/Hamiltonian pencil. Let $\lambda_{1}, \ldots, \lambda_{m}$ be the pairwise distinct, finite, purely imaginary eigenvalues of $\lambda S-H$ with algebraic multiplicities $p_{1}, \ldots, p_{m}$ and let $p_{\infty}$ be the algebraic multiplicity of the eigenvalue $\infty$. Then the following statements are equivalent.

1. There exists a nonsingular matrix $P \in \mathbb{C}^{2 n \times 2 n}$ such that

$$
-J P^{*} J(\lambda S-H) P=\lambda\left[\begin{array}{cc}
S_{11} & S_{12} \\
0 & S_{11}^{*}
\end{array}\right]-\left[\begin{array}{cc}
H_{11} & H_{12} \\
0 & -H_{11}^{*}
\end{array}\right],
$$

where $S_{11}$ and $H_{11}$ are upper triangular.

2. There is a unitary matrix $Q \in \mathbb{C}^{2 n \times 2 n}$ such that

$$
-J Q^{*} J(\lambda S-H) Q=\lambda\left[\begin{array}{cc}
\hat{S}_{11} & \hat{S}_{12} \\
0 & \hat{S}_{11}^{*}
\end{array}\right]-\left[\begin{array}{cc}
\hat{H}_{11} & \hat{H}_{12} \\
0 & -\hat{H}_{11}^{*}
\end{array}\right],
$$

where $\hat{S}_{11}$ and $\hat{H}_{11}$ are upper triangular. 
3. If the columns of $U_{k} \in \mathbb{C}^{2 n \times p_{k}}, k=1, \ldots, m$, form a basis of the deflating subspace associated with $\lambda_{k}$, then

$$
U_{k}^{*} J S U_{k} \sim_{c} J_{p_{k} / 2}
$$

Furthermore, if $p_{\infty} \neq 0$ and if the columns of $U_{\infty} \in \mathbb{C}^{2 n \times p_{\infty}}$ form a basis of the deflating subspace associated with the eigenvalue $\infty$, then

$$
U_{\infty}^{*} J H U_{\infty} \sim_{c} i J_{p_{\infty} / 2}
$$

Proof. $1 \Rightarrow 2$ : If $P \in \mathbb{C}^{2 n \times 2 n}$ is nonsingular such that (31) holds, Lemma 17 implies that there exists a factorization (30). Using this we obtain from (31), that

$$
\begin{aligned}
& -J Q^{*} J(\lambda S-H) Q \\
= & J\left[\begin{array}{cc}
R_{11} & R_{12} \\
0 & R_{22}^{*}
\end{array}\right]^{-*} J\left(\lambda\left[\begin{array}{cc}
S_{11} S_{12} \\
0 & S_{11}^{*}
\end{array}\right]-\left[\begin{array}{cc}
H_{11} & H_{12} \\
0 & -H_{11}^{*}
\end{array}\right]\right)\left[\begin{array}{cc}
R_{11} & R_{12} \\
0 & R_{22}^{*}
\end{array}\right] \\
= & \lambda\left[\begin{array}{cc}
-R_{22}^{-1} S_{11} R_{11}^{-1} & * \\
0 & -R_{11}^{-*} S_{11}^{*} R_{22}^{-*}
\end{array}\right]-\left[\begin{array}{cc}
-R_{22}^{-1} H_{11} R_{11}^{-1} & * \\
0 & R_{11}^{-*} H_{11}^{*} R_{22}^{-*}
\end{array}\right],
\end{aligned}
$$

where the upper left subpencil $-R_{22}^{-1}\left(\lambda S_{11}-H_{11}\right) R_{11}^{-1}$ is still upper triangular.

$2 \Rightarrow 3$ : Let $\lambda S-H$ be in the form (32). First we show that the pencil is (up to the permutation of rows and columns) $J$-congruent to a direct sum of skew-Hamiltonian/Hamiltonian pencils in the form (32) that have only one pair $\left(\mu_{j},-\mu_{j}^{*}\right)$ of eigenvalues. Therefore, consider a $4 \times 4$ subpencil

$$
\lambda \breve{S}-\breve{H}=\lambda\left[\begin{array}{cccc}
s_{11} & s_{12} & s_{13} & s_{14} \\
0 & s_{22} & -s_{14}^{*} & s_{24} \\
0 & 0 & s_{11}^{*} & 0 \\
0 & 0 & s_{12}^{*} & s_{22}^{*}
\end{array}\right]-\left[\begin{array}{cccc}
h_{11} & h_{12} & h_{13} & h_{14} \\
0 & h_{22} & h_{14}^{*} & h_{24} \\
0 & 0 & -h_{11}^{*} & 0 \\
0 & 0 & -h_{12}^{*} & -h_{22}^{*}
\end{array}\right]
$$

of the pencil in (32) such that $\lambda s_{11}-h_{11}$ has the eigenvalue $\lambda_{0}$ and such that $\lambda s_{22}-h_{22}$ has the eigenvalue $\mu_{0}$. Furthermore, let $\lambda_{0} \neq \mu_{0},-\mu_{0}^{*}$. Next we show that the (1,2)-elements of $\check{S}$ and $\check{H}$, respectively, can be simultaneously set to zero. Therefore, let

$$
P=\left[\begin{array}{llll}
1 & x & 0 & 0 \\
0 & 1 & 0 & 0 \\
0 & 0 & 1 & 0 \\
0 & 0 & y & 1
\end{array}\right]
$$

where $x, y \in \mathbb{C}$, and consider $-J P^{*} J \check{S} P$ and $-J P^{*} J \check{H} P$. If we want to set the $(1,2)$ elements of both matrices to zero, we have to solve the equations

$$
\text { and } \begin{aligned}
s_{12}+s_{22} y^{*}+s_{11} x & =0 \\
h_{12}+h_{22} y^{*}+h_{11} x & =0 .
\end{aligned}
$$


This implies $\left(h_{22} s_{11}-h_{11} s_{22}\right) x=s_{22} h_{12}-h_{22} s_{12}$. Since $\lambda_{0} \neq \mu_{0}$, it follows that

$$
h_{22} s_{11}-h_{11} s_{22} \neq 0 \text {. }
$$

Therefore, $x$ (and $y$ ) can be chosen such that the $(1,2)$-elements of both $-J P^{*} J \hat{S} P$ and $-J P^{*} J \hat{H} P$ are zero. In an analogous way we show that using a $J$-congruence transformation with the matrix

$$
P=\left[\begin{array}{llll}
1 & 0 & 0 & x \\
0 & 1 & y & 0 \\
0 & 0 & 1 & 0 \\
0 & 0 & 0 & 1
\end{array}\right]
$$

the (1,4)-elements of $\check{S}$ and $\check{H}$ can also be simultaneously set to zero. Here, we need $\lambda_{0} \neq-\mu_{0}^{*}$. (We note that this transformation has no effect on the $2 \times 2$ diagonal blocks of $\lambda \check{S}-\check{H}$.) Now it is clear that a sequence of $J$-congruence transformations can be used to obtain a pencil that is (up to the permutation of rows and columns) a direct sum of skew-Hamiltonian/Hamiltonian pencils in the form (32) such that each pencil has only one pair $\left(\mu_{j},-\mu_{j}^{*}\right)$ of eigenvalues, and we may consider the blocks separately.

Furthermore, we may assume w.l.o.g. that the bases $U_{k}$ and $U_{\infty}$ are canonical, for if (33) holds for a special basis $U_{k}$ it holds for every basis of the deflating subspace associated with $\lambda_{k}$, because a change of basis means the transition from $U_{k}$ to $U_{k} X$, where $X \in \mathbb{C}^{p_{k} \times p_{k}}$ is nonsingular. (The same argument holds for $U_{\infty}$.) Thus, if

$$
\lambda \tilde{S}-\tilde{H}=\lambda\left[\begin{array}{cc}
\tilde{S}_{11} & \tilde{S}_{12} \\
0 & \tilde{S}_{11}^{*}
\end{array}\right]-\left[\begin{array}{cc}
\tilde{H}_{11} & \tilde{H}_{12} \\
0 & -\tilde{H}_{11}^{*}
\end{array}\right]
$$

is a skew-Hamiltonian/Hamiltonian pencil satisfying (31) and that has only one eigenvalue that is finite and purely imaginary, it remains to show that $J \tilde{S}$ is congruent to $J$. This also proves (34), for we can reduce the case of the eigenvalue $\infty$ to the case of the eigenvalue zero by considering $\lambda(i \tilde{H})-(i \tilde{S})$ according to Lemma 2.3. Now we obtain

$$
\begin{aligned}
& {\left[\begin{array}{cc}
\tilde{S}_{11}^{-1} & -\frac{1}{2} \tilde{S}_{11}^{-1} \tilde{S}_{12} \\
0 & I
\end{array}\right]^{*} J \tilde{S}\left[\begin{array}{cc}
\tilde{S}_{11}^{-1} & -\frac{1}{2} \tilde{S}_{11}^{-1} \tilde{S}_{12} \\
0 & I
\end{array}\right] } \\
= & {\left[\begin{array}{cc}
\tilde{S}_{11}^{-*} & 0 \\
-\frac{1}{2} \tilde{S}_{12}^{*} \tilde{S}_{11}^{-*} & I
\end{array}\right]\left[\begin{array}{cc}
0 & \tilde{S}_{11}^{*} \\
-\tilde{S}_{11}-\tilde{S}_{12}
\end{array}\right]\left[\begin{array}{cc}
\tilde{S}_{11}^{-1} & -\frac{1}{2} \tilde{S}_{11}^{-1} \tilde{S}_{12} \\
0 & I
\end{array}\right] } \\
= & {\left[\begin{array}{cc}
0 & I \\
-I & 0
\end{array}\right]=J }
\end{aligned}
$$

i.e., $J \tilde{S}$ is congruent to $J$.

$3 \Rightarrow 1$ : Since (33) and (34) imply that all the algebraic multiplicities of the purely imaginary eigenvalues are even, it is by Theorem 11 and Lemmas 128 again sufficient to consider the case that $\lambda S-H$ has only one eigenvalue that is finite and purely imaginary. 
Since $J S$ is congruent to $J$ we have that $S$ is $J$-congruent to the identity matrix. Thus there exists $P_{1} \in \mathbb{C}^{2 n \times 2 n}$ nonsingular such that

$$
-J P_{1}^{*} J(\lambda S-H) P_{1}=\lambda I-\hat{H},
$$

where $\hat{H}$ is a Hamiltonian matrix having only one eigenvalue, which is purely imaginary. Thus, $\hat{H}$ trivially satisfies (28) and by Theorem 15 we find a symplectic matrix $P_{2} \in \mathbb{C}^{2 n \times 2 n}$, i.e., $P_{2}^{-1}=-J P_{2}^{*} J$, such that $P_{2}^{-1} \hat{H} P_{2}$ is in $J$-Schur form (26). We obtain (31) by setting $P=P_{1} P_{2}$.

We call a pencil of the form (31) a pencil in $J$-Schur form.

\section{An almost-Schur form under $J$-congruence}

We have seen in section 4 that not every skew-Hamiltonian/Hamiltonian pencil can be reduced to a $J$-Schur form by unitary $J$-congruence transformation. Thus, if we want to obtain a condensed form from which the eigenvalues can be read off in a simple way, we have to use also nonunitary $J$-congruence transformations. In this section we will present a condensed form that we will call almost-Schur form and that can be obtained by applying $J$-congruence transformations, where $P$ in (8) is a product of unitary or almost unitary matrices.

Definition 19 Let $P=\left[p_{1}, \ldots, p_{n}\right] \in \mathbb{C}^{n}$ be nonsingular. Then $P$ is called almost unitary if there exists an index $k$ such that $\left(p_{1}, \ldots, p_{k-1}, p_{k+1}, \ldots, p_{n}\right)$ is an orthonormal tuple of vectors.

Theorem 20 Let $\lambda S-H \in \mathbb{C}^{2 n \times 2 n}$ be a regular skew-Hamiltonian/Hamiltonian pencil. Then there exists a nonsingular matrix $P \in \mathbb{C}^{2 n \times 2 n}$ and $k \in \mathbb{N}$ such that

$$
\begin{aligned}
& -J P^{*} J(\lambda S-H) P \\
= & \lambda\left[\begin{array}{cccc}
S_{11} & S_{12} & S_{13} & S_{14} \\
0 & 0 & -S_{14}^{*} & S_{24} \\
0 & 0 & S_{11}^{*} & 0 \\
0 & S_{42} & S_{12}^{*} & 0
\end{array}\right]-\left[\begin{array}{cccc}
H_{11} & H_{12} & H_{13} & H_{14} \\
0 & 0 & H_{14}^{*} & H_{24} \\
0 & 0 & -H_{11}^{*} & 0 \\
0 & H_{42} & -H_{12}^{*} & 0
\end{array}\right],
\end{aligned}
$$

where the following conditions hold:

1. $\lambda S_{11}-H_{11} \in \mathbb{C}^{k \times k}$ is an upper triangular pencil having only eigenvalues with nonnegative real part.

2. $S_{24}, S_{42}, H_{24}, H_{42} \in \mathbb{C}^{(n-k) \times(n-k)}$ are diagonal and all the eigenvalues of the skewHamiltonian/Hamiltonian pencil

$$
\lambda\left[\begin{array}{cc}
0 & S_{24} \\
S_{42} & 0
\end{array}\right]-\left[\begin{array}{cc}
0 & H_{24} \\
H_{42} & 0
\end{array}\right]
$$

are purely imaginary. 
3. The spectrum of $\lambda S-H$ is equal to the union of the spectra of

$$
\lambda\left[\begin{array}{cc}
S_{11} & S_{13} \\
0 & S_{11}^{*}
\end{array}\right]-\left[\begin{array}{cc}
H_{11} & H_{13} \\
0 & -H_{11}^{*}
\end{array}\right] \text { and } \lambda\left[\begin{array}{cc}
0 & S_{24} \\
S_{42} & 0
\end{array}\right]-\left[\begin{array}{cc}
0 & H_{24} \\
H_{42} & 0
\end{array}\right] .
$$

4. $P$ is a product of unitary and almost unitary matrices.

Proof. We consider two cases:

(a) Let us assume that there exists an eigenvector $x \neq 0$ associated with an eigenvalue $\lambda_{0}$ of $\lambda S-H$ such that

$$
x^{*} J S x=0 \text { and } x^{*} J H x=0 .
$$

Since $\lambda S-H$ is regular, we have $J S x \neq 0$ or $J H x \neq 0$. Assume that $J S x \neq 0$; i.e., $\lambda_{0} \neq \infty$ (otherwise the argument proceeds analogously by exchanging $J S x$ and $J H x$ ). Then (36) implies in particular that $x$ and $J S x$ are linearly independent. Let $\left(q_{2}, \ldots, q_{n}, q_{n+2}, \ldots, q_{2 n}\right)$ be an orthonormal basis of $\operatorname{Span}(x, J S x)^{\perp}$. We assume that $\operatorname{Re}\left(\lambda_{0}\right) \geq 0$ and set

$$
Q:=\left[x, q_{2}, \ldots, q_{n}, J S x, q_{n+2}, \ldots, q_{2 n}\right] .
$$

(In the case $\operatorname{Re}\left(\lambda_{0}\right)<0$ we set $Q=\left[J S x, q_{2}, \ldots, q_{n}, x, q_{n+2}, \ldots, q_{2 n}\right]$ and the argument proceeds analogously.)

Note that (after having normalized $x$ and $J S x$ ) the matrix $Q$ is unitary by (36) and by definition of the $q_{i}$. We obtain

$$
-J Q^{*} J S Q e_{1}=\left[\begin{array}{c}
-(J S x)^{*} J S x \\
0 \\
\vdots \\
0
\end{array}\right]=\left[\begin{array}{c}
-x^{*} S^{*} S x \\
0 \\
\vdots \\
0
\end{array}\right]
$$

and

$$
-J Q^{*} J H Q e_{1}=\left[\begin{array}{c}
-(J S x)^{*} J H x \\
0 \\
\vdots \\
0
\end{array}\right]=\left[\begin{array}{c}
-x^{*} S^{*} H x \\
0 \\
\vdots \\
0
\end{array}\right] .
$$

Since $-J Q^{*} J(\lambda S-H) Q$ is still skew-Hamiltonian/Hamiltonian, we obtain

$$
\begin{aligned}
& -J Q^{*} J(\lambda S-H) Q \\
= & \lambda\left[\begin{array}{cccc}
s_{11} & * & * & * \\
0 & S_{22} & * & S_{24} \\
0 & 0 & s_{11}^{*} & 0 \\
0 & S_{42} & * & S_{22}^{*}
\end{array}\right]-\left[\begin{array}{cccc}
h_{11} & * & * & * \\
0 & H_{22} & * & H_{24} \\
0 & 0 & -h_{11}^{*} & 0 \\
0 & H_{42} & * & -H_{22}^{*}
\end{array}\right],
\end{aligned}
$$

where $S_{22}, S_{24}, S_{42}, H_{22}, H_{24}, H_{42} \in \mathbb{C}^{(n-1) \times(n-1)}, s_{11}=-x^{*} S^{*} S x$, and $h_{11}=-x^{*} S^{*} H x$. Obviously the pencil $\lambda s_{11}-h_{11}$ has the eigenvalue $\lambda_{0}$ and by permutation of rows and columns we see that the spectrum of $\lambda S-H$ is equal to the union of the spectra of

$$
\lambda\left[\begin{array}{cc}
s_{11} & * \\
0 & s_{11}^{*}
\end{array}\right]-\left[\begin{array}{cc}
h_{11} & * \\
0 & -h_{11}^{*}
\end{array}\right] \text { and } \lambda\left[\begin{array}{l}
S_{22} S_{24} \\
S_{42} S_{22}^{*}
\end{array}\right]-\left[\begin{array}{cc}
H_{22} & H_{24} \\
H_{42} & -H_{22}^{*}
\end{array}\right] .
$$


(b) If the assumption in (a) does not hold, then for every eigenvector $x \neq 0$ associated with an eigenvalue of $\lambda S-H$ we have

$$
x^{*} J S x \neq 0 \text { or } \quad x^{*} J H x \neq 0 .
$$

In this case we see by Lemma 9 that all the eigenvalues of $\lambda S-H$ are purely imaginary and by Lemma 10 they all have partial multiplicities equal to one. Once again we assume $x^{*} J S x \neq 0$. (Otherwise the argument proceeds analogously by exchanging $J S x$ and $J H x$.) Let $\left(q_{1}, \ldots, q_{n-1}, q_{n+1}, \ldots, q_{2 n}\right)$ be an orthonormal basis of $\operatorname{Span}(J S x)^{\perp}$. Then

$$
P_{1}:=\left[q_{1}, \ldots, q_{n-1}, x, q_{n+1}, \ldots, q_{2 n}\right]
$$

is invertible. This follows, since if $\alpha_{1}, \ldots, \alpha_{2 n} \in \mathbb{C}$ are such that

$$
\alpha_{1} q_{1}+\cdots+\alpha_{n-1} q_{n-1}+\alpha_{n} x+\alpha_{n+1} q_{n+1}+\cdots+\alpha_{2 n} q_{2 n}=0,
$$

then multiplying (40) from the left by $x^{*} S^{*} J$ we obtain $\alpha_{2 n}=0$, since $x^{*} J S x \neq 0$, and therefore $\alpha_{i}=0$ for all $i=1, \ldots, 2 n$, since the $q_{i}$ form a basis. Hence we obtain

$$
-J P_{1}^{*} J S x=\left[\begin{array}{c}
0 \\
\vdots \\
0 \\
s_{42}
\end{array}\right] \text { and }-J P_{1}^{*} J H x=\left[\begin{array}{c}
0 \\
\vdots \\
0 \\
h_{42}
\end{array}\right] \text {, }
$$

where $s_{42}=x^{*} J S x$ and $h_{42}=x^{*} J H x$. This implies that

$$
\begin{aligned}
& -J P_{1}^{*} J(\lambda S-H) P_{1} \\
= & \lambda\left[\begin{array}{cccc}
S_{11} & 0 & S_{13} & S_{14} \\
S_{21} & 0 & -S_{14}^{*} & S_{24} \\
S_{31} & 0 & S_{11}^{*} & S_{21}^{*} \\
0 & s_{42} & 0 & 0
\end{array}\right]-\left[\begin{array}{cccc}
H_{11} & 0 & H_{13} & H_{14} \\
H_{21} & 0 & H_{14}^{*} & H_{24} \\
H_{31} & 0 & -H_{11}^{*} & -H_{21}^{*} \\
0 & h_{42} & 0 & 0
\end{array}\right],
\end{aligned}
$$

where $S_{11}, H_{11} \in \mathbb{C}^{(n-1) \times(n-1)}$ and the other blocks have corresponding sizes. Now set

$$
\lambda \tilde{S}-\tilde{H}:=\lambda\left[\begin{array}{ccc}
S_{11} & S_{13} & S_{14} \\
S_{21} & -S_{14}^{*} & S_{24} \\
S_{31} & S_{11}^{*} & S_{21}^{*}
\end{array}\right]-\left[\begin{array}{ccc}
H_{11} & H_{13} & H_{14} \\
H_{21} & H_{14}^{*} & H_{24} \\
H_{31} & -H_{11}^{*} & -H_{21}^{*}
\end{array}\right]
$$

and let $y$ be an eigenvalue of $\lambda \tilde{S}-\tilde{H}$ associated with the eigenvalue $\mu_{0}$. By (38) we find

$$
y^{*} J \tilde{S} y \neq 0 \quad \text { or } \quad y^{*} J \tilde{H} y \neq 0,
$$

which implies, in particular, that also $\mu_{0}$ is purely imaginary with partial multiplicity equal to one. Again we assume $y^{*} J \tilde{S} y \neq 0$ and choose an orthonormal basis $\left(p_{1}, \ldots, p_{2 n-2}\right)$ of $\operatorname{Span}(J \tilde{S} x)^{\perp}$. Analogous to the proof of the nonsingularity of (39), we can show that the 
vectors $y$ and $\left(p_{1}, \ldots, p_{2 n-2}\right)$ are linearly independent. Embedding these vectors canonically in $\mathbb{C}^{n}$ and then setting

$$
P_{2}:=\left[p_{1}, \ldots, p_{n-1}, e_{n}, p_{n}, \ldots, p_{2 n-2}, y\right]
$$

we obtain

$$
\begin{aligned}
& -J P_{2}^{*} P_{1}^{*} J(\lambda S-H) P_{1} P_{2} \\
= & \lambda\left[\begin{array}{cccc}
\tilde{S}_{11} & 0 & \tilde{S}_{13} & 0 \\
0 & 0 & 0 & s_{24} \\
\tilde{S}_{31} & 0 & \tilde{S}_{11}^{*} & 0 \\
0 & s_{42} & 0 & 0
\end{array}\right]-\left[\begin{array}{cccc}
\tilde{H}_{11} & 0 & \tilde{H}_{13} & 0 \\
0 & 0 & 0 & h_{24} \\
\tilde{H}_{31} & 0 & -\tilde{H}_{11}^{*} & 0 \\
0 & h_{42} & 0 & 0
\end{array}\right],
\end{aligned}
$$

where $\tilde{S}_{11}, \tilde{S}_{13}, \tilde{S}_{31}, \tilde{H}_{11}, \tilde{H}_{13}, \tilde{H}_{31} \in \mathbb{C}^{(n-1) \times(n-1)}, s_{24}=y^{*} J \tilde{S} y$, and $h_{24}=y^{*} J \tilde{H} y$. Thus, the spectrum of $\lambda S-H$ is equal to the union of the spectra of

$$
\lambda\left[\begin{array}{cc}
\tilde{S}_{11} & \tilde{S}_{13} \\
\tilde{S}_{31} & \tilde{S}_{11}^{*}
\end{array}\right]-\left[\begin{array}{cc}
\tilde{H}_{11} & \tilde{H}_{13} \\
\tilde{H}_{31} & -\tilde{H}_{11}^{*}
\end{array}\right] \text { and } \lambda\left[\begin{array}{cc}
0 & s_{24} \\
s_{42} & 0
\end{array}\right]-\left[\begin{array}{cc}
0 & h_{24} \\
h_{42} & 0
\end{array}\right],
$$

and the eigenvalues of $\lambda\left[\begin{array}{cc}0 & s_{24} \\ s_{42} & 0\end{array}\right]-\left[\begin{array}{cc}0 & h_{24} \\ h_{42} & 0\end{array}\right]$ are $\lambda_{0}$ and $\mu_{0}$.

In both cases we have reduced the problem to a problem of smaller dimension and the proof follows by induction.

Remark 21 The matrices $-J P^{*} J S P$ and $-J P^{*} J H P$ in (35) have the pattern

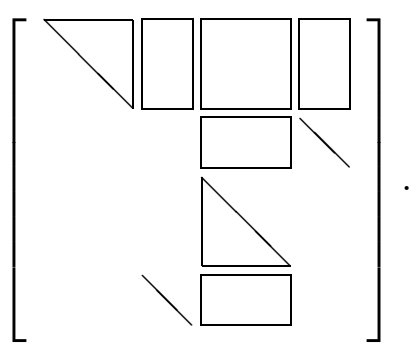

Remark 22 The proof of Theorem 20 also suggests a procedure to compute the almostSchur form (35). But during the computation the following difficulties may occur:

1. In the first step we may compute only one eigenvalue and then reduce the pencil as indicated in part (a) or (b) of the proof of Theorem 20. This means that if we compute an eigenvector satisfying (38), we do not know whether (38) holds for all the eigenvalues or if there exists an eigenvalue such that (36) holds. In particular we might fail in finding an eigenvector of the subpencil (42) that satisfies (38). Consider, for example, the pencil

$$
\lambda\left[\begin{array}{cccc}
0 & 0 & i & i \\
i & 0 & i & 0 \\
0 & 0 & 0 & -i \\
0 & -i & 0 & 0
\end{array}\right]-\left[\begin{array}{cccc}
0 & 0 & 1 & 2 \\
1 & 0 & 2 & 0 \\
0 & 0 & 0 & -1 \\
0 & 1 & 0 & 0
\end{array}\right]
$$


The subpencil

$$
\lambda\left[\begin{array}{ccc}
0 & i & i \\
i & i & 0 \\
0 & 0 & -i
\end{array}\right]-\left[\begin{array}{ccc}
0 & 1 & 2 \\
1 & 2 & 0 \\
0 & 0 & -1
\end{array}\right]
$$

has the eigenvalue $i$ with partial multiplicity 3. Thus, Lemma 10 implies that we do not find an eigenvector satisfying (38). Therefore, the following observation may be helpful.

Given a skew-Hamiltonian/Hamiltonian pencil in the form (41) and an eigenvector $x$ satisfying (36), we can perform a step as indicated in part (a) of the proof of Theorem 20 by choosing the basis of $\operatorname{Span}(x, J S x)^{\perp}$ (resp., $\left.\operatorname{Span}(x, J H x)^{\perp}\right)$ such that $q_{n}$ in (37) is equal to $e_{n}$. This is always possible, since $e_{n}$ is an eigenvector. Then we obtain by Lemmas 9 or 10 that

$$
e_{n}^{*} J S x=0
$$

and by the special structure of the pencil (41) also

$$
e_{n}^{*} x=0
$$

Hence we can proceed reducing the matrix as indicated in (a) until we find another eigenvector $y$ satisfying (38).

2. If we compute an eigenvector satisfying (38) during the reduction of $\lambda S-H$, we do not achieve the $J$-Schur form by proceeding as indicated in part (b) of the proof of Theorem 20. But this does in general not mean that there is no $J$-Schur form. Consider as an example the skew-Hamiltonian matrix

$$
S:=\left[\begin{array}{cccc}
-1-0.5 i & 0 & 0.5 i & 0 \\
0 & -1-0.5 i & 0 & 0.5 i \\
-0.5 i & 0 & -1+0.5 i & 0 \\
0 & -0.5 i & 0 & -1+0.5 i
\end{array}\right]
$$

and the skew-Hamiltonian/Hamiltonian pencil $\lambda S-(i S)$. We note that $e_{1}$ and $e_{3}$ are eigenvectors associated with the eigenvalue $i$. Now we have

$$
e_{1}^{*} J S e_{1} \neq 0 \quad \text { and } \quad e_{3}^{*} J S e_{3} \neq 0
$$

but

$$
\left(e_{1}+e_{3}\right)^{*} J S\left(e_{1}+e_{3}\right)=0=\left(e_{1}+e_{3}\right)^{*} J H\left(e_{1}+e_{3}\right) .
$$

Thus, if we choose the eigenvector $e_{1}$ instead of $\left(e_{1}+e_{3}\right)$ for the reduction of the pencil, we would create the almost-Schur form, although there exists a $J$-Schur form. Setting

$$
U:=\frac{1}{\sqrt{2}} J\left[\begin{array}{cccc}
1 & 0 & -1 & 0 \\
0 & 1 & 0 & -1 \\
1 & 0 & 1 & 0 \\
0 & 1 & 0 & 1
\end{array}\right]
$$


we obtain

$$
\begin{gathered}
-J U^{*} J(\lambda S-i S) U \\
=-\lambda\left[\begin{array}{cccc}
1 & 0 & -i & 0 \\
0 & 1 & 0 & -i \\
0 & 0 & 1 & 0 \\
0 & 0 & 0 & 1
\end{array}\right]+\left[\begin{array}{cccc}
i & 0 & 1 & 0 \\
0 & i & 0 & 1 \\
0 & 0 & i & 0 \\
0 & 0 & 0 & i
\end{array}\right] .
\end{gathered}
$$

Since we do not know how to compute an eigenvector satisfying (36), if it exists, we currently see no method to avoid this problem.

In the real case we obtain a result similar to Theorem 20.

Definition 23 Let $P=\left[p_{1}, \ldots, p_{n}\right] \in \mathbb{R}^{n}$ be nonsingular. Then $P$ is called almost orthogonal if there exists indices $k, l$ such that

$$
\left(p_{1}, \ldots, p_{k-1}, p_{k+1}, \ldots, p_{l-1}, p_{l+1}, \ldots, p_{n}\right)
$$

is an orthonormal tuple of vectors.

Theorem 24 Let $\lambda S-H \in \mathbb{R}^{2 n \times 2 n}$ be a regular skew-Hamiltonian/Hamiltonian pencil. Then there exists a nonsingular matrix $P \in \mathbb{R}^{2 n \times 2 n}$ and $k \in \mathbb{N}$ such that

$$
\begin{aligned}
& -J P^{*} J(\lambda S-H) P \\
= & \lambda\left[\begin{array}{cccc}
S_{11} & S_{12} & S_{13} & S_{14} \\
0 & 0 & -S_{14}^{*} & S_{24} \\
0 & 0 & S_{11}^{*} & 0 \\
0 & S_{42} & S_{12}^{*} & 0
\end{array}\right]-\left[\begin{array}{cccc}
H_{11} & H_{12} & H_{13} & H_{14} \\
0 & 0 & H_{14}^{*} & H_{24} \\
0 & 0 & -H_{11}^{*} & 0 \\
0 & H_{42} & -H_{12}^{*} & 0
\end{array}\right],
\end{aligned}
$$

where the following conditions hold:

1. $\lambda S_{11}-H_{11} \in \mathbb{R}^{k \times k}$ is a quasi upper triangular pencil having only eigenvalues with nonnegative real part.

2. The pencils $\lambda S_{24}-H_{24}, \lambda S_{42}-H_{42} \in \mathbb{R}^{(n-k) \times(n-k)}$ are block diagonal and the diagonal blocks are either of the form

$$
\lambda\left[\begin{array}{ccc}
0 & & \\
& \ddots & \\
& & 0
\end{array}\right]-\left[\begin{array}{lll}
1 & & \\
& \ddots & \\
& & 1
\end{array}\right] \in \mathbb{R}^{m \times m}
$$

for $m \in \mathbb{N}$ or of the form

$$
\lambda\left[\begin{array}{rr}
0 & s \\
-s & 0
\end{array}\right]-\left[\begin{array}{ll}
h & 0 \\
0 & h
\end{array}\right],
$$

where $s, h \in \mathbb{R} \backslash\{0\}$. In particular, all the eigenvalues of the skew-Hamiltonian/Hamiltonian pencil

$$
\lambda\left[\begin{array}{cc}
0 & S_{24} \\
S_{42} & 0
\end{array}\right]-\left[\begin{array}{cc}
0 & H_{24} \\
H_{42} & 0
\end{array}\right]
$$

are nonzero and purely imaginary. 
3. The spectrum of $\lambda S-H$ is equal to the union of the spectra of

$$
\lambda\left[\begin{array}{cc}
S_{11} & S_{13} \\
0 & S_{11}^{*}
\end{array}\right]-\left[\begin{array}{cc}
H_{11} & H_{13} \\
0 & -H_{11}^{*}
\end{array}\right] \text { and } \lambda\left[\begin{array}{cc}
0 & S_{24} \\
S_{42} & 0
\end{array}\right]-\left[\begin{array}{cc}
0 & H_{24} \\
H_{42} & 0
\end{array}\right] .
$$

4. $P$ is a product of orthogonal and almost orthogonal matrices.

Proof. Analogous to the proof of Theorem 20 we distinguish two cases.

(a) Let us assume that there exists an eigenvector $x \in \mathbb{C}^{n} \backslash\{0\}$ associated with an eigenvalue $\lambda_{0} \in \mathbb{C}$ of $\lambda S-H$ such that

$$
x^{*} J S x=0 \text { and } x^{*} J H x=0 .
$$

If $\lambda_{0}$ and $x$ are real we can proceed as in the proof of Theorem 20. So let us assume that $\lambda_{0}$ has nonzero real part and that $x=y+i z$, where $y, z \in \mathbb{R}^{n}$. Furthermore we assume once again w.l.o.g. that $J S x \neq 0$. (Otherwise the argument proceeds analogously by exchanging $J S x$ and $J H x$.) This means in particular that both $J S y$ and $J S z$ do not vanish. Otherwise $y$ or $z$ would be an eigenvector associated with the eigenvalue $\infty$. It is well known that $y, z$ form a basis of a two-dimensional subspace; i.e., in particular $y$ and $z$ are linearly independent. Furthermore, we have by (48) that

$$
\begin{aligned}
& y^{*} J S y+z^{*} J S z+i\left(y^{*} J S z-z^{*} J S y\right) \\
= & (y+i z)^{*} J S(y+i z)=0 .
\end{aligned}
$$

Since $\lambda_{0}^{*} \neq-\lambda_{0}^{*}$ and $y-i z$ is an eigenvalue associated with $\lambda_{0}^{*}$, we also have by Lemma 9 that

$$
\begin{aligned}
& y^{*} J S y-z^{*} J S z+i\left(y^{*} J S z-z^{*} J S y\right) \\
= & (y-i z)^{*} J S(y+i z)=0 .
\end{aligned}
$$

Hence by adding, resp., subtracting (49) and (50) we obtain

$$
y^{*} J S y=z^{*} J S z=z^{*} J S y=y^{*} J S z=0 .
$$

If we choose a real orthonormal basis $\left(r_{1}, r_{2}\right)$ of $\operatorname{Span}(y, z)$, we still have

$$
r_{1}, r_{2} \perp J S r_{1}, J S r_{2}
$$

Now let $\left(q_{1}, \ldots, q_{2 n-4}\right)$ be a real orthonormal basis of $\operatorname{Span}\left(r_{1}, r_{2}, J S r_{1}, J S r_{2}\right)$. Then (assuming again w.l.o.g. that $\lambda_{0}$ has nonnegative real part and after having normalized $r_{1}, r_{2}, J S r_{1}$, and $\left.J S r_{2}\right)$ the matrix

$$
Q:=\left[r_{1}, r_{2}, q_{1}, \ldots, q_{n-2}, J S r_{1}, J S r_{2}, q_{n-1}, \ldots, q_{2 n-4}\right]
$$


is orthogonal and we obtain

$$
\begin{aligned}
& -J Q^{*} J(\lambda S-H) Q \\
= & \lambda\left[\begin{array}{cccc}
S_{11} & * & * & * \\
0 & S_{22} & * & S_{24} \\
0 & 0 & S_{11}^{*} & 0 \\
0 & S_{42} & * & S_{22}^{*}
\end{array}\right]-\left[\begin{array}{cccc}
H_{11} & * & * & * \\
0 & H_{22} & * & H_{24} \\
0 & 0 & -H_{11}^{*} & 0 \\
0 & H_{42} & * & -H_{22}^{*}
\end{array}\right],
\end{aligned}
$$

where $S_{11}, H_{11} \in \mathbb{R}^{2 \times 2}$ and $S_{22}, S_{24}, S_{42}, H_{22}, H_{24}, H_{42} \in \mathbb{R}^{(n-2) \times(n-2)}$. Obviously the pencil $\lambda S_{11}-H_{11}$ has the eigenvalues $\lambda_{0}$ and $\lambda_{0}^{*}$ and the spectrum of $\lambda S-H$ is equal to the union of the spectra of

$$
\lambda\left[\begin{array}{cc}
S_{11} & * \\
0 & S_{11}^{*}
\end{array}\right]-\left[\begin{array}{cc}
H_{11} & * \\
0 & -H_{11}^{*}
\end{array}\right] \text { and }\left[\begin{array}{l}
S_{22} S_{24} \\
S_{42} S_{22}^{*}
\end{array}\right]-\left[\begin{array}{cc}
H_{22} & H_{24} \\
H_{42} & -H_{22}^{*}
\end{array}\right] .
$$

(b) If the assumption in (a) does not hold, then for every eigenvector $x \in \mathbb{C}^{n} \backslash\{0\}$ associated with an eigenvalue of $\lambda S-H$ we have

$$
x^{*} J S x \neq 0 \text { or } x^{*} J H x \neq 0 .
$$

This means again that all the eigenvalues of $\lambda S-H$ are purely imaginary and all their partial multiplicities are one. In addition we have that $\lambda S-H$ cannot have the eigenvalue zero, for if $w \in \mathbb{R}^{n}$ is an eigenvector associated with zero, i.e., $H w=0$, since $S$ is skewHamiltonian, we obtain

$$
w^{*} J S w=\left(w^{*} J S w\right)^{*}=w^{*} S^{*} J w=-w^{*} J S w ;
$$

i.e., $w^{*} J S w=0=w^{*} J H w$. In the case of the eigenvalue $\infty$, we can find a real eigenvector and so we proceed as in the proof of Theorem 20. Thus it remains to consider the case that $x$ is an eigenvector associated with the eigenvalue $i \mu$ for a $\mu \in \mathbb{R} \backslash\{0\}$ and that $x=y+i z$, where $y, z \in \mathbb{R}^{n}$. Again $y$ and $z$ are linearly independent and we have both $J S y \neq 0$ and $J S z \neq 0$. Now let $\left(q_{1}, \ldots, q_{n-2}, q_{n+1}, \ldots, q_{2 n}\right)$ be a real orthonormal basis of $\operatorname{Span}(J S y, J S z)^{\perp}$. Analogous to the proof of the nonsingularity of (39) we can show that

$$
\tilde{P}:=\left[q_{1}, \ldots, q_{n-2}, y, z, q_{n+1}, \ldots, q_{2 n}\right]
$$

is nonsingular. We obtain

$$
\begin{aligned}
& -J \tilde{P}^{*} J(\lambda S-H) \tilde{P} \\
= & \lambda\left[\begin{array}{cccc}
S_{11} & 0 & S_{13} & S_{14} \\
S_{21} & 0 & -S_{14}^{*} & S_{24} \\
S_{31} & 0 & S_{11}^{*} & S_{21}^{*} \\
0 & S_{42} & 0 & 0
\end{array}\right]-\left[\begin{array}{cccc}
H_{11} & 0 & H_{13} & H_{14} \\
H_{21} & 0 & H_{14}^{*} & H_{24} \\
H_{31} & 0 & -H_{11}^{*} & -H_{21}^{*} \\
0 & H_{42} & 0 & 0
\end{array}\right],
\end{aligned}
$$

where

$$
S_{42}=\left[\begin{array}{ll}
y^{*} J S y & y^{*} J S z \\
z^{*} J S y & z^{*} J S z
\end{array}\right] \text { and } H_{42}=\left[\begin{array}{ll}
y^{*} J H y & y^{*} J H z \\
z^{*} J H y & z^{*} J H z
\end{array}\right]
$$


and $S_{11}, H_{11} \in \mathbb{R}^{(n-2) \times(n-2)}$. The other blocks have corresponding sizes. Since $S_{42}$ is skew-symmetric and $y^{*} J S z=-z^{*} J S y$ we obtain

$$
S_{42}=\left[\begin{array}{cc}
0 & s \\
-s & 0
\end{array}\right]
$$

where $s:=-y^{*} J S z$. This yields in particular that $y^{*} J S y=0$. On the other hand we have $i \mu S(y+i z)=H(y+i z)$; i.e.,

$$
-\mu S z=H y \quad \text { and } \quad \mu S y=H z .
$$

Therefore, we obtain that

$$
y^{*} J H z=\mu y^{*} J S y=0
$$

and

$$
y^{*} J H y=-\mu y^{*} J S z=\mu z^{*} S^{*} J y=\mu z^{*} J S y=z^{*} J H z .
$$

Thus,

$$
H_{42}=\left[\begin{array}{ll}
h & 0 \\
0 & h
\end{array}\right]
$$

where $h:=-y^{*} J H y$. The rest of the proof of (b) follows analogous to the proof of Theorem 20 , reducing the subpencil

$$
\lambda\left[\begin{array}{ccc}
S_{11} & S_{13} & S_{14} \\
S_{21} & -S_{14}^{*} & S_{24} \\
S_{31} & S_{11}^{*} & S_{21}^{*}
\end{array}\right]-\left[\begin{array}{ccc}
H_{11} & H_{13} & H_{14} \\
H_{21} & H_{14}^{*} & H_{24} \\
H_{31} & -H_{11}^{*} & -H_{21}^{*}
\end{array}\right]
$$

by choosing a nonreal eigenvector associated with an eigenvalue $i \tilde{\mu}, \tilde{\mu} \in \mathbb{R}$, or two real eigenvectors associated with the eigenvalue $\infty$. (Note that the eigenvalue $\infty$ must have even algebraic multiplicity, since all the other eigenvalues of the pencil occur in pairs and the size of the pencil is even.)

In both cases we have reduced the problem to a problem of smaller dimension and the proof follows by induction.

Lemma 25 Let $\lambda S-H$ be a real skew-Hamiltonian/Hamiltonian pencil. Then the following conditions hold:

1. Every block of odd size belonging to the eigenvalue zero in the Kronecker canonical form occurs with even multiplicity.

2. Every block of even size belonging to the eigenvalue $\infty$ in the Kronecker canonical form occurs with even multiplicity.

Proof. See the proof in [20] of the analogous result for pencils $\lambda A-B$, where $A$ is symmetric and $B$ is skew symmetric. 


\section{Conclusions}

We have discussed canonical forms and almost-Schur forms for skew-Hamiltonian/Hamiltonian pencils under $J$-congruence. Also a necessary and sufficient condition for the existence of a $J$-Schur form has been given.

\section{Acknowledgment}

We thank V. Mehrmann for helpful discussions and for initiating this research.

\section{References}

[1] H. Braun and M. Koecher, Jordan-Algebren, Springer-Verlag, Berlin, Heidelberg, New York, 1966.

[2] N. Burgoyne and R. Cushman, Normal Forms for Real Hamiltonian Matrices, in The 1976 Ames Research Center Conference on Geometric Control Theory, C. Martin and R. Hermann, editors, Mathematical Sciences Press, Brookline, MA, 1976, pp. 483-529.

[3] A. Ciampi, Classical Hamiltonian linear systems, Queen's papers in pure and applied mathematics, 31 (1972).

[4] A. Ciampi, Classification of Hamiltonian linear systems, Indiana U. Math. J., 23 (1973), pp 513-526.

[5] D.-Ž Djoković and J. Patera and P. Winterwitz and H. Zassenhaus, Normal forms of elements of classical real and complex Lie and Jordan algebras, J. Math. Phys.,24 (1983), pp. 1363-1374.

[6] A. Ferrante And B.-C. Levy, Canonical form of symplectic matrix pencils, Linear Algebra Appl., 274 (1998), pp. 259-300.

[7] F. Gantmacher, Theory of Matrices, Vol. 1, Chelsea, New York, 1959.

[8] F. Gantmacher, Theory of Matrices, Vol. 2, Chelsea, New York, 1959.

[9] G. Golub and C. Van Loan, Matrix Computations, Johns Hopkins University Press, Baltimore, 1983.

[10] P. Lancaster and L. Rodman, Algebraic Riccati Equations, Clarendon Press, Oxford, 1995.

[11] A.-J. Laub and K. Meyer, Canonical forms for symplectic and Hamiltonian matrices, Celestial Mech., 9 (1974), pp. 213-238. 
[12] W.-W. Lin And T.-C. Ho, On Schur type decompositions for Hamiltonian and Symplectic Pencils, Tech. report, Institute of Applied Mathematics, National Tsing Hua University, Taiwan, 1990.

[13] V. Mehrmann, The Autonomous Linear Quadratic Control Problem, Theory and Numerical Solution, Lecture Notes in Control and Information Sciences 163, SpringerVerlag, Heidelberg, July 1991.

[14] V. Mehrmann, A step towards a unified treatment of continuous and discrete time control problems, Linear Algebra Appl., 241-243 (1996), pp. 749-779.

[15] V. Mehrmann and H. Xu, Canonical Forms for Hamiltonian and Symplectic Matrices and Pencils, Preprint SFB 393/98-07, Sonderforschungsbereich 393 Numerische Simulation auf massiv parallelen Rechnern, Fakultät für Mathematik, Technische Universität Chemnitz, D-09107 Chemnitz, FRG, 1998.

[16] K. Meyer and G. Hall, Introduction to Hamiltonian Dynamical Systems and the N-Body Problem, Springer, New York, 1992.

[17] C. Paige And C. VAn LoAn, A Schur decomposition for Hamiltonian matrices, Linear Algebra Appl., 14 (1981), pp. 11-32.

[18] I. Sminnov, Linear Algebra and Group Theory, McGraw-Hill, New York, Toronto, London, 1961.

[19] R. Thompson, The characteristic polynomial of a principal subpencil of a hermitian matrix pencil, Linear Algebra Appl., 14 (1976), pp. 135-177.

[20] R. Thompson, Pencils of complex and real symmetric and skew matrices, Linear Algebra Appl., 147 (1991), pp. 323-371.

[21] H. Weyl, The Classical Groups, Princeton University Press, Princeton, NJ, 1946.

[22] H.K. Wimmer, Normal forms of symplectic pencils and discrete-time algebraic Riccati Equations, Linear Algebra Appl., 147 (1991), pp. 411-440. 